\title{
Probiotic and Oxytocin Combination Therapy in Patients with Autism Spectrum Disorder: A Randomized, Double-Blinded, Placebo-Controlled Pilot Trial
}

\author{
Xue-Jun Kong ${ }^{1,2, *}$, Jun Liu ${ }^{1,3}{ }^{3}$, Kevin Liu ${ }^{1}{ }^{1}$, Madelyn Koh ${ }^{1}$, Hannah Sherman ${ }^{1}$, Siyu Liu ${ }^{1}$, Ruiyi Tian ${ }^{1}$, \\ Piyawat Sukijthamapan ${ }^{3}$, Jiuju Wang ${ }^{1}$, Michelle Fong ${ }^{1}{ }^{\mathbb{D}}$, Lei Xu ${ }^{3,4}$, Cullen Clairmont ${ }^{1}$, Min-Seo Jeong ${ }^{1}$, \\ Alice Li ${ }^{1}$, Maria Lopes ${ }^{1}$, Veronica Hagan ${ }^{1}$, Tess Dutton ${ }^{1}$, Suk-Tak (Phoebe) Chan ${ }^{1}$, Hang Lee ${ }^{3,5}$, Amy Kendall ${ }^{1}$, \\ Kenneth Kwong ${ }^{1}$ and Yiqing Song ${ }^{6} \mathbb{C}$
}

check for

updates

Citation: Kong, X.-J.; Liu, J.; Liu, K.; Koh, M.; Sherman, H.; Liu, S.; Tian, R.; Sukijthamapan, P.; Wang, J.; Fong, M.; et al. Probiotic and Oxytocin Combination Therapy in Patients with Autism Spectrum Disorder: A Randomized, Double-Blinded, Placebo-Controlled Pilot Trial. Nutrients 2021, 13, 1552. https:// doi.org/10.3390/nu13051552

Received: 9 April 2021

Accepted: 2 May 2021

Published: 5 May 2021

Publisher's Note: MDPI stays neutral with regard to jurisdictional claims in published maps and institutional affiliations.

Copyright: (c) 2021 by the authors. Licensee MDPI, Basel, Switzerland. This article is an open access article distributed under the terms and conditions of the Creative Commons Attribution (CC BY) license (https:// creativecommons.org/licenses/by/ $4.0 /)$.
1 Athinoula A. Martinos Center, Massachusetts General Hospital, Charlestown, MA 02129, USA; Jun_Liu@hms.harvard.edu (J.L.); kliu16@mgh.harvard.edu (K.L.); mkoh@mgh.harvard.edu (M.K.); htsherman@mgh.harvard.edu (H.S.); SLIU41@mgh.harvard.edu (S.L.); RTIAN2@mgh.harvard.edu (R.T.); Jwang106@mgh.harvard.edu (J.W.); Michelle_Fong@DFCI.HARVARD.EDU (M.F.); CCLAIRMONT1@mgh.harvard.edu (C.C.); msjeong@mgh.harvard.edu (M.-S.J.); CLI36@mgh.harvard.edu (A.L.); MLOPES6@mgh.harvard.edu (M.L.); VHAGAN@mgh.harvard.edu (V.H.); tdutton1@bwh.harvard.edu (T.D.); stchan@mgh.harvard.edu (S.-T.C.); alkendall@mgh.harvard.edu (A.K.); KKWONG@mgh.harvard.edu (K.K.)

2 Department of Psychiatry, Beth Israel Deaconess Medical Center, Boston, MA 02215, USA

3 Harvard Medical School, Boston, MA 02115, USA; Piyawat_Sukijthamapan@DFCI.harvard.edu (P.S.); lei@steele.mgh.harvard.edu (L.X.); hlee5@mgh.harvard.edu (H.L.)

4 Department of Radiation Oncology, Massachusetts General Hospital, Boston, MA 02114, USA

5 MGH Biostatistics Center, Massachusetts General Hospital, Boston, MA 02114, USA

6 Department of Epidemiology, Indiana University, Richard M. Fairbanks School of Public Health, Indianapolis, IN 46202, USA; yiqsong@iu.edu

* Correspondence: xkong1@mgh.harvard.edu; Tel.: +1-(617)-726-9439

Abstract: Autism spectrum disorder (ASD) is a rapidly growing neurodevelopmental disorder. Both probiotics and oxytocin were reported to have therapeutic potential; however, the combination therapy has not yet been studied. We conducted a randomized, double-blinded, placebo-controlled, 2-stage pilot trial in 35 individuals with ASD aged 3-20 years (median $=10.30$ years). Subjects were randomly assigned to receive daily Lactobacillus plantarum PS128 probiotic $\left(6 \times 10^{10} \mathrm{CFUs}\right)$ or a placebo for 28 weeks; starting on week 16, both groups received oxytocin. The primary outcomes measure socio-behavioral severity using the Social Responsiveness Scale (SRS) and Aberrant Behavior Checklist (ABC). The secondary outcomes include measures of the Clinical Global Impression (CGI) scale, fecal microbiome, blood serum inflammatory markers, and oxytocin. All outcomes were compared between the two groups at baseline, 16 weeks, and 28 weeks into treatment. We observed improvements in ABC and SRS scores and significant improvements in CGI-improvement between those receiving probiotics and oxytocin combination therapy compared to those receiving placebo $(p<0.05)$. A significant number of favorable gut microbiome network hubs were also identified after combination therapy $(p<0.05)$. The favorable social cognition response of the combination regimen is highly correlated with the abundance of the Eubacterium hallii group. Our findings suggest synergic effects between probiotics PS128 and oxytocin in ASD patients, although further investigation is warranted.

Keywords: autism spectrum disorder (ASD); probiotics; oxytocin; microbiome; inflammation markers

\section{Introduction}

Autism spectrum disorder (ASD) is a complex neurodevelopmental disorder featuring impaired social communication and stereotypical repetitive behavioral patterns. ASD has become a serious health issue due to its rapidly rising prevalence. According to a recent 
report from the CDC, the prevalence of ASD has risen to 1 in 54 children [1]. However, its etiology remains elusive, and effective treatment is still largely unavailable.

Gut microbiome composition and inflammation have been reported to be involved in the pathogenesis of ASD through the gut-brain axis [2]. Recent evidence demonstrates that alterations in the gut microbiota of ASD individuals changes both gastrointestinal (GI) physiology and behaviors via the gut-microbiome-brain axis [3,4]. Probiotic varieties used in both animal studies and clinical trials have demonstrated efficacy in improving ASD core symptoms [5,6]. Evidence that probiotics have the potential to improve neuropsychiatric symptoms via the gut-brain axis is not limited to ASD. In fact, there is an entire subgroup of probiotics, known as psychobiotics, that may provide health benefits in patients with psychiatric illness [7]. Animal studies have shown some psychobiotic strains can improve depression-like behavior [8], anxiety-like behavior [9], cognition [10], and autism-like behaviors, such as communication defect and stereotypic behaviors [11]. One psychobiotic, Lactobacillus plantarum PS128 (PS128), showed ameliorative effects on depression- and anxiety-like behaviors in different mouse models [12,13]. When administered to children with ASD, PS128 was shown to improve anxiety, rule-breaking behaviors, and hyperactivity/impulsivity [5].

Oxytocin (OXT), a neuropeptide produced by the hypothalamus, is well known for its ability to modulate emotional and social communication, bonding, and reward-related behaviors [14]. OXT signaling is implicated in the etiology of ASD, as previous studies using OXT receptor knockout mouse models exhibit autistic-like behavior, such as deficits in social interaction [15]. Subsequent studies have shown that OXT treatment enhanced sociability in two mouse models of ASD [16]. OXT shows promising therapeutic potential for ASD core symptoms because it can be easily administered and can work as a costeffective treatment with minimal adverse effects. Furthermore, OXT plays an important role in the gut-brain axis and is likely inducible by certain probiotics such as Lactobacillus reuteri [14]. However, potential biological connections between Lactobacillus plantarum, including PS128, and endogenous OXT have not been studied. Moreover, the interactions between these two promising interventions, OXT, and PS128 have not been tested.

We designed this double-blinded, randomized, placebo-controlled, two-stage pilot trial to test our hypothesis that combination therapy with probiotics and OXT results in a therapeutic synergy that exerts beneficial effects on ASD symptoms. We simultaneously measured the clinical index for ASD core symptoms, gut microbiome profile, and levels of OXT and inflammatory markers in the blood to evaluate the efficacy of the combination therapy and identify impacting factors with predictive value for treatment outcomes.

\section{Materials and Methods}

\subsection{Trial Design}

This clinical trial is a randomized, double-blind, and placebo-controlled study in accordance with the Consolidated Standards of Reporting Trials (CONSORT) guidelines. Subjects were randomized to two groups with a 1:1 ratio into this two-stage study. To achieve a statistical power of $80 \%$ for primary outcomes with a large effect size of 0.8 (Cohen's d) assumed, a total of 60 participants (30 in each arm) were required. However, as we are primarily interested in studying the preliminary effects of the proposed treatment, we enrolled and randomized 35 subjects who were included in the data analysis. In stage 1 , the probiotics group received oral probiotics PS128 while the placebo group received oral placebo for 16 weeks. In stage 2, both groups continued their respective administration and simultaneously added intranasal oxytocin spray. The treatment proceeded for a total of 28 weeks with 3 visits for outcomes measurement at 0,16 weeks, and 28 weeks (V1, $\mathrm{V} 2$, and V3, respectively). While we originally planned to conduct the study outcome measurements at weeks 0,12 , and 24 , we decided to prolong the study treatment for stage 1 for an additional 4 weeks based on some preliminary results. Such a change was justified by our determination that prolonged treatment of probiotic supplementation with the current strain of interest has not been previously investigated [5]. 
This study was conducted according to the guidelines described in the Declaration of Helsinki. Ethical approval of this study was issued by the Internal Review Board (IRB) of Massachusetts General Hospital (2017P001667). The clinical trial was registered through ClinicalTrials.gov (accessed on 2 May 2021) with identifier NCT03337035. Written informed consent was obtained from competent adult subjects or from the parents or legal guardians of children and adults with cognitive impairment according to the Internal Review Board (IRB) requirements. The protocol of this study was published previously [6].

Compliance and safety assessments of potential adverse effects were assessed monthly via telephone check-in and self-report via Internet Research Electronic Data Capture (REDCap, v9.5.23) software. All adverse events were reported to the Human Research Committee of Massachusetts General Hospital promptly in accordance with guidelines. The Data and Safety Monitoring Plan (DSMP) was in place and approved by IRB to ensure the safety of participants, the validity of data, and the appropriate termination of this study.

\subsection{Participants}

Study participants were recruited through advertising posters/flyers in local communities and through ASD parent networks and workshops. Participants were included if they were 3-25 years old and had a pre-existing diagnosis of ASD confirmed by the Diagnostic and Statistical Manual of Mental Disorder (DSM-IV TR/-5) criteria, Autism Diagnostic Observation Schedule, Second Edition (ADOS-2), and/or The Autism Diagnostic Interview-Revised (ADI-R). Other inclusion criteria are: participants must have stable medications for at least 4 weeks, have no planned changes in medications or psychosocial interventions during the trial period, are willing to provide stool samples and blood in the timely manner, and are willing to participate in interviews and study procedures. A potential participant was excluded if the subject was pregnant (before or during the study); had comorbidity of other neurological and/or psychiatric disorders, such as bipolar disorders or history of a substance use disorder; was on psychotropic medications; had an active cardiovascular disease that is not controlled by medication; or had received oxytocin or probiotic treatment within the last 4 weeks. The participants were interviewed and tested in the private room of the clinical research setting of Athinoula A. Martinos Center at Massachusetts General Hospital.

\subsection{Randomization and Blinding}

Randomization and allocation concealment were performed by a statistician who was not part of the research team, in collaboration with the Massachusetts General Hospital research pharmacy. Randomization sampling numbers were electronically generated, and central randomization at the research pharmacy using coded drug containers that are identical in appearance were prepared by the pharmacy to ensure allocation concealment. Blinding was maintained by making the capsules look identical. Both participants and the research staff who collected the outcome data were blinded to treatment status.

\subsection{Interventions}

Lactobacillus plantarum PS128 (PS128), which was isolated from a traditional Taiwan fermented mustard food [17], was deposited under DSMZ Accession No. DSM 28632. The genome architecture of PS128 was illustrated [18]. Both animal and human studies with PS128 demonstrated great safety $[5,12,13,19]$. The probiotic capsule contained only PS128 as a single-strain probiotic. Dosage in the study was 2 capsules a day $\left(6 \times 10^{10} \mathrm{CFUs}\right)$. Microcrystalline cellulose capsules were used as a placebo for PS128. Both probiotics and placebo capsules were free gifts obtained from Bened Biomedical Co., Ltd (Taipei, Taiwan). In this study, oxytocin was administered nasally. The Syntocinon ${ }^{\circledR}$ Spray (Novartis Pharma AG; purchased from Apotheke Roter Ochsen, Schaffhausen, Switzerland and Victoria Apotheke Wholesale, Schwerzenbach, Switzerland) is currently the most commonly used standardized oxytocin nasal spray for clinical trials worldwide. We instructed the patient and family members about the use of this spray. Dosing began with 1 puff of 4 IU 
daily for the first week of the second stage. Subsequently, the dosage was increased to 1 puff per nostril daily $(8 \mathrm{IU} / \mathrm{d})$ for the second week and 1 puff per nostril twice a day $(16 \mathrm{IU} / \mathrm{d})$ for the third week. The dosage was then titrated up to the maximum dose of $32 \mathrm{IU}$ daily, which is 2 puffs per nostril twice a day, starting on the fourth week. The dosage of $32 \mathrm{IU}$ per day has been approved as safe and adequate in even younger patients (age 3-8 years) by a previous publication [20]. Another study reported a 4-week intranasal OXT treatment (24 IU, twice daily with total $48 \mathrm{IU}$ per day, which is more than the max dose in this study of $32 \mathrm{IU}$ per day) in 32 children with ASD, aged 6-12 years old [21]. We achieved an active IND from the FDA, and the IND number is 138827 for Syntocinon ${ }^{\circledR}$ (Pitocin, Oxytocin).

\subsection{Outcomes}

\subsubsection{Primary Outcome Measures}

We evaluated two primary outcome measures:

1. Change in caregiver-rated Social Responsiveness Scale (SRS) [22];

2. Change in caregiver-rated Aberrant Behavior Checklist (ABC) [23].

The SRS is used to assess social interest and interaction based on five subscales. We interviewed all subjects older than 4 years. The $\mathrm{ABC}$ is an informant rating instrument that was empirically derived by a principal component analysis. It contains 58 items that resolve onto five subscales. We interviewed all of the subjects older than 5 years.

\subsubsection{Secondary Outcome Measures}

- Blood sample collection and circulating biomarker analysis

Participants presented to the Athinoula A. Martinos Center for the visit after an $8 \mathrm{~h}$ fast three times (week 0, week 16, and week 28). Blood was drawn and processed to obtain serum, labelled with a unique code, and stored at $-80^{\circ} \mathrm{C}$. Circulating serum OXT, myelin basic protein (MBP), glial fibrillary acidic protein (GFAP), S100 calcium-binding protein B (S100B), and interleukin-1 $\beta$ (IL-1 $\beta$ ) were measured by ELISA (R\&D Systems Inc., Minneapolis, MN, USA), following the manufacturer's instructions.

- GI symptom severity assessments

GI symptoms were assessed by the validated GI severity index (GSI), including constipation, diarrhea, stool consistency, stool smell, flatulence, abdominal pain, unexpected daytime irritability, night-time awakening, and abdominal tenderness. The stool status was scored using the Bristol Stool Chart.

- Clinical Global Impression (CGI)

The clinical global impression (CGI) scale was developed for use in clinical trials to provide a brief, stand-alone assessment of the clinician's view of the patient's global functioning changes with the study medication. The CGI comprises two companion oneitem measures evaluating the following: (a) severity of psychopathology from 1 to 7 (CGI-S) and (b) the improvement or change from the initiation of treatment on a similar seven-point scale (CGI-I) [24].

\subsection{Stool Sample Processing}

Stool samples were collected with an OMNIgene Gut OMR-200 collection kit (DNA Genotek Inc.) by the participants at home under the supervision of trained parents and stored at room temperature, before de-identification and delivery or shipment to the Athinoula A. Martinos Center, where stool samples were stored at $-80^{\circ} \mathrm{C}$ freezer. After all of the experiment samples were collected after week 28 , they were hand delivered with dry ice packaging to a laboratory at Brigham \& Woman's Hospital for DNA extraction and sequencing analysis.

Microbial DNA was then extracted according to the manufacturer's instructions, and DNA samples were quantified with a NanoDrop spectrophotometer. A260/A280 
ratios were also measured to confirm high-purity DNA yield. Microbial 16S rRNA V4 genomic regions from total gut DNA samples were amplified with the following primers: 515F (AATGATACGGCGACCACCGAGATCTACACNNNNNNNNTATGGTAATTGTGTGCCAGCMGCCGCGGTAA) and 806R (CAAGCAGAAGACGGCATACGAGATNNNNNNNNAGTCAGTCAGCCGGACTACHVGGGTWTCTAAT). PCR products were purified and analyzed using a Bioanalyzer DNA kit, followed by quantification with real-time PCR. DNA libraries were pooled and sequenced on an Illumina MiSeq next-generation sequencing system (Illumina; San Diego, CA, USA) using a V4 $2 \times 250$ bp paired-end protocol with overlapping reads.

\subsection{Statistical Analysis}

Data analyses were performed based on the intention-to-treat principle. The primary outcomes for the treatment comparisons were the changes in the scores of SRS and ABC (SRS T-score, ABC T-score). The secondary outcomes measurement includes CGI, GSI, levels of serum markers, and the gut microbiome.

An independent sample $t$-test/Wilcoxon rank-sum test for continuous variables was used to detect between-group differences in the measurement changes over the intervention course. Paired sample $t$-test and Wilcoxon signed-rank test were used to test the withingroup difference in the primary outcomes and secondary outcomes before and after the intervention (V2-V1 and V3-V1). The z-test for equality of proportions without continuity correction was applied to differences in the proportion of subjects displaying change in secondary outcome measures.

We additionally performed a stratified analysis based on baseline SRS/ABC score, GI condition, and neuroinflammation/neuro-injury serum marker levels.

Sequencing data were processed and analyzed with a QIIME 2 [25], and $\alpha$-diversity was calculated by Chao-1, Faith PD, Evenness, and observed OTUs using the Phyloseq R package. $\beta$-diversity, weighted UniFrac, unweighted UniFrac, Bray-Curtis, and Jaccard were analyzed.

SparCC (Sparse Correlations for Compositional data) co-abundance networks were constructed to examine the longitudinal change in associations between gut microbiota [26]. Correlations with magnitudes greater than a SparCC cutoff of 0.7 were considered significant. Identified hub taxa and the respective hub scores are indicated by the size of the circle. False discovery rate (FDR)-based type 1 error control was made per study visit on a group-wise basis across all assessed variables. This was conducted via MaAsLin2 for assessed correlations between primary and secondary outcomes and blood serum marker concentrations against microbiota relative abundances [27]. Significant correlations were considered at an FDR $<0.1$.

PICRUSt (phylogenetic investigation of communities by reconstruction of unobserved states) is a computational approach to predict the functional composition of a metagenome using marker gene data and a database of reference genomes and was applied to the current $16 \mathrm{~S}$ dataset. The relative change in abundance of each feature abundance (ASVs or pathways) between visits V1 and V2 (V2-V1) and visits V1 and V3 (V3-V1) were computed for each experimental subject. Then, differential analyses were performed on the relative changes between the probiotics and placebo groups with Wilcoxon rank-sum test.

\section{Results}

\subsection{Demographics}

The flowchart of the study is shown in Figure 1. Between 12 December 2018 and June 17, 2019, we enrolled and randomized 35 patients with ASD aged 3-20 years (median $=10.30$ years; 26 males, 9 females). The placebo group subjects had an age range of 4.69-19.70 years (10.70 \pm 4.76 years; 11 males, 6 females), while the probiotic group subjects had an age range of 3.60-18.50 years (9.85 \pm 4.91 years; 15 males, 3 females). The baseline demographic features and clinical indices of the 35 participants are summarized in Table 1. ASD severity measures via ABC, SRS, and CGI-S of all participants suggest 
that the baseline severity is determined to be $275 \pm 32.3$ via the $A B C$ standardized score (T-score), $82.6 \pm 11.6$ via the SRS standardized score (T-score), and $5.11 \pm 1.02$ via the CGI-S. Group-wise comparisons of such scores are also summarized in Table 1. There were no significant differences between the two groups in these demographic and clinical indices $(p>0.05)$. No serious or severe adverse events were observed. One subject was terminated due to minor nose bleeding in stage 2 that resolved quickly on its own; this subject had a history of recurrent nose bleeding related to their seasonal rhinitis. Another subject was terminated due to oral ambulatory antibiotics use for a mild upper respiratory infection. Other self-withdrawals were due to moving, travel, or other administrative reasons which were found to have no relation to the study or any adverse events. There was no significant difference of dropouts found between the two groups $(p>0.05)$.

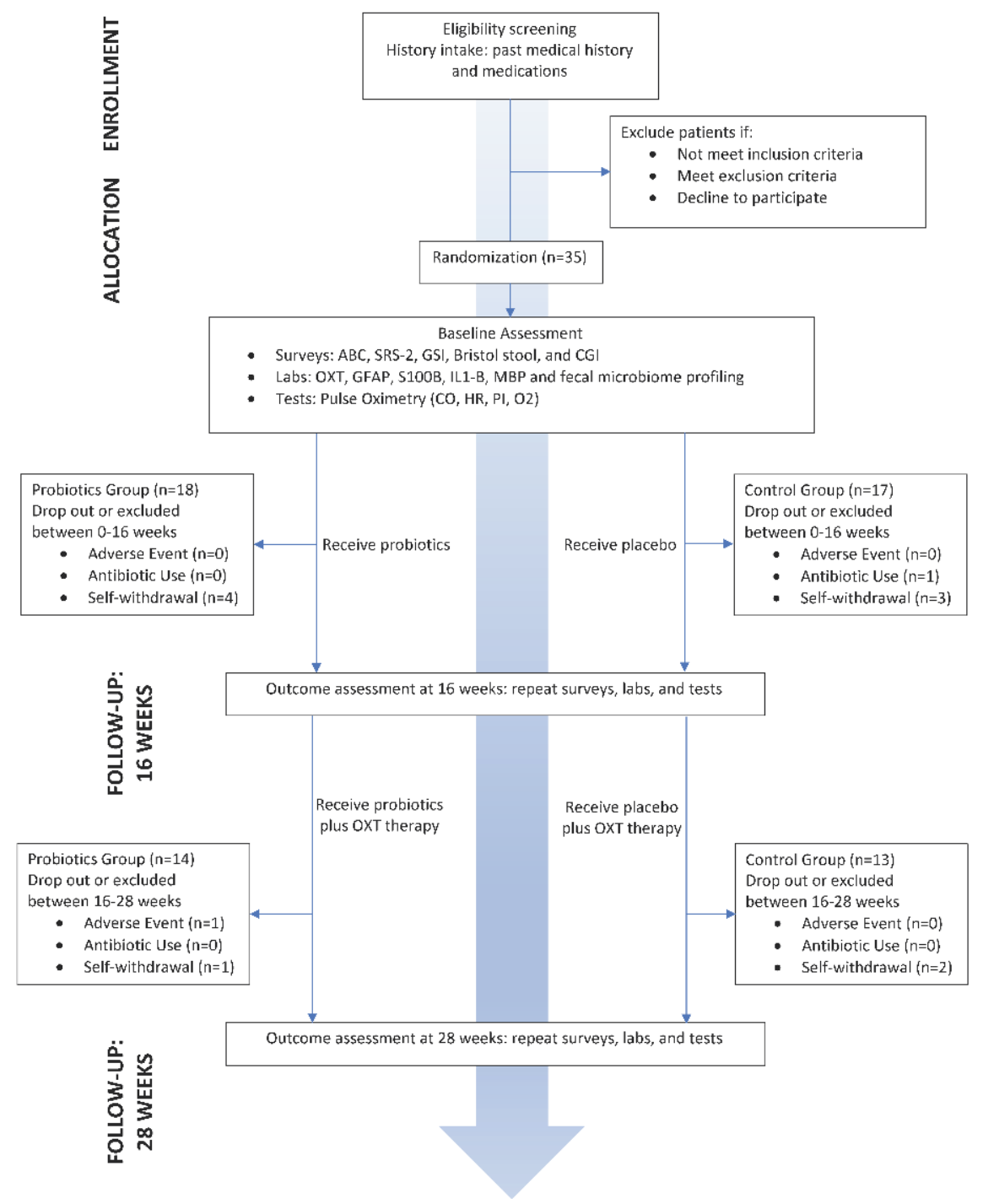

Figure 1. Flowchart of overall study design and conduct.

\subsection{Socio-Behavioral Parameters and Other Clinical Indices}

Changes in socio-behavioral parameters as measured by ABC and SRS from visit 1 to visit 2 (V2-V1) for the control group and probiotics group, and from visit 1 to visit 3 (V3-V1) for the OXT group and the probiotic + OXT combination group (Table 2). We performed independent Wilcoxon rank-sum tests for subjects in each treatment group against the control group subjects. Trends of improvement in the total ABC score $(p=0.077)$, stereotypic behavior score $(p=0.069)$, and SRS cognition score $(p=0.059)$ were observed in the combination therapy group (Probiotic + OXT), although no significant differences 
were observed in the total scores or subscales of the ABC and SRS (Wilcoxon rank-sum test, $p>0.05)$.

Table 1. Summary of subject demographics and clinical indices at baseline.

\begin{tabular}{|c|c|c|c|}
\hline & $\begin{array}{c}\text { Placebo } \\
(n=17)\end{array}$ & $\begin{array}{l}\text { Probiotic } \\
(n=18)\end{array}$ & $p$-Value * \\
\hline \multicolumn{4}{|l|}{ Demographic } \\
\hline \multirow{2}{*}{$\begin{array}{l}\text { Age }(\text { Mean } \pm \text { SD }) \\
\text { Sex }(N, \%)\end{array}$} & $10.7 \pm 4.76$ & $9.85 \pm 4.91$ & 0.66 \\
\hline & & & \\
\hline Male & $11(64.7 \%)$ & $15(83.3 \%)$ & \\
\hline Female & $6(35.3 \%)$ & $3(16.7 \%)$ & 0.38 \\
\hline \multicolumn{4}{|l|}{ Ethnicity $(N, \%)$} \\
\hline Asian & $14(82.4 \%)$ & $14(77.8 \%)$ & \\
\hline Hispanic & 0 & $1(5.5 \%)$ & 0.62 \\
\hline White & $3(17.6 \%)$ & $3(16.7 \%)$ & \\
\hline \multicolumn{4}{|l|}{ Clinical Indices } \\
\hline GI Severity Index (Mean \pm SD) & $3.33 \pm 1.37$ & $2.54 \pm 2.03$ & 0.18 \\
\hline \multicolumn{4}{|l|}{ Stool Type (Bristol stool chart; $N, \%$ ) } \\
\hline Type $1 \& 2$ (Constipated) & $1(5.9 \%)$ & $1(5.6 \%)$ & \\
\hline Type $3 \& 4$ (Normal) & $12(70.6 \%)$ & $13(72.2 \%)$ & 1.00 \\
\hline Type 5, 6, 7 (Loose Stool) & $3(17.6 \%)$ & $3(16.7 \%)$ & \\
\hline ABC Standardized Score (T-score, Mean \pm SD) & $278 \pm 34.8$ & $272 \pm 30.2$ & 0.38 \\
\hline SRS Standardized Score (T-score, Mean \pm SD) & $83.0 \pm 12.1$ & $82.3 \pm 11.5$ & 0.96 \\
\hline CGI-S (Mean \pm SD) & $5.12 \pm 1.17$ & $5.11 \pm 0.90$ & 0.97 \\
\hline
\end{tabular}

* Continuous data was evaluated for $p$-values via the Wilcoxon rank-sum test while categorical data was evaluated for intergroup differences via the Pearson's $\chi^{2}$-test with Yates' continuity correction.

Table 2. Summary of improvement in socio-behavioral measures. Data are presented as mean change \pm SD.

\begin{tabular}{|c|c|c|c|c|c|c|c|}
\hline & \multicolumn{4}{|c|}{ Improvement in Score (Mean Change \pm SD) } & \multicolumn{3}{|c|}{$p$-Value * } \\
\hline & Control & Probiotic & OXT & $\begin{array}{l}\text { Probiotic }+ \\
\text { OXT }\end{array}$ & Probiotic & OXT & $\begin{array}{c}\text { Probiotic }+ \\
\text { OXT }\end{array}$ \\
\hline \multicolumn{8}{|l|}{$\mathrm{ABC}$} \\
\hline Total Score & $15.00 \pm 26.75$ & $6.67 \pm 26.00$ & $12.33 \pm 23.16$ & $-10.43 \pm 31.91$ & 0.48 & 0.84 & 0.077 \\
\hline Irritability (S1) & $3.45 \pm 6.67$ & $-0.92 \pm 6.20$ & $2.17 \pm 3.97$ & $-2.43 \pm 9.86$ & 0.19 & 0.84 & 0.20 \\
\hline Social Withdrawal (S2) & $1.82 \pm 8.30$ & $3.50 \pm 6.36$ & $1.67 \pm 7.92$ & $-4.00 \pm 10.26$ & 0.46 & 1 & 0.28 \\
\hline Stereotypic Behavior (S3) & $3.18 \pm 4.38$ & $1.17 \pm 6.64$ & $1.83 \pm 5.78$ & $-1.29 \pm 4.27$ & 0.67 & 0.45 & 0.069 \\
\hline Hyperactivity/Noncompliance & $5.64 \pm 8.31$ & $2.33 \pm 8.91$ & $5.50 \pm 7.45$ & $-1.57 \pm 10.50$ & 0.34 & 0.84 & 0.16 \\
\hline Inappropriate Speech (S5) & $0.91 \pm 2.12$ & $0.58 \pm 1.78$ & $1.67 \pm 3.31$ & $-1.14 \pm 3.02$ & 0.66 & 0.80 & 0.20 \\
\hline \multicolumn{8}{|l|}{ SRS } \\
\hline Total Score & $22.09 \pm 23.71$ & $12.31 \pm 22.21$ & $10.00 \pm 24.71$ & $4.88 \pm 22.95$ & 0.45 & 0.23 & 0.26 \\
\hline Awareness & $1.18 \pm 2.36$ & $1.15 \pm 2.58$ & $1.33 \pm 2.58$ & $0.13 \pm 3.04$ & 0.86 & 0.96 & 0.28 \\
\hline Cognition & $4.73 \pm 4.54$ & $0.92 \pm 4.31$ & $2.00 \pm 5.18$ & $0.38 \pm 5.53$ & 0.15 & 0.15 & 0.059 \\
\hline Communication & $7.09 \pm 9.45$ & $4.46 \pm 7.13$ & $0.50 \pm 9.09$ & $2.00 \pm 8.11$ & 0.68 & 0.11 & 0.36 \\
\hline Motivation & $3.27 \pm 3.58$ & $2.54 \pm 4.96$ & $2.83 \pm 5.67$ & $0.88 \pm 6.15$ & 0.77 & 0.61 & 0.16 \\
\hline Mannerisms & $5.82 \pm 6.82$ & $3.23 \pm 6.61$ & $3.33 \pm 6.09$ & $1.50 \pm 5.50$ & 0.58 & 0.39 & 0.20 \\
\hline
\end{tabular}

* Provided $p$-values are based on Wilcoxon rank-sum test between the mean improvement in score in the control group and the respective treatment groups.

CGI was assessed to evaluate ASD symptoms and the relative extent of improvement in symptoms at each visiting time. As seen in Figure 2, the proportion of subjects showing improvement is significantly increased only in the probiotic + OXT combination group when compared against that of the control group (Pearson's $\chi^{2}$-test, $p<0.05$ ), while the changes in the probiotics or OXT alone groups were non-significant, though a trend of improvement was observed in both intervention groups. The waterfall data of the CGI score reduction in each group is shown in supplement Supp. Figure S1. 
Subjects with at least minimal improvement in CGI-I

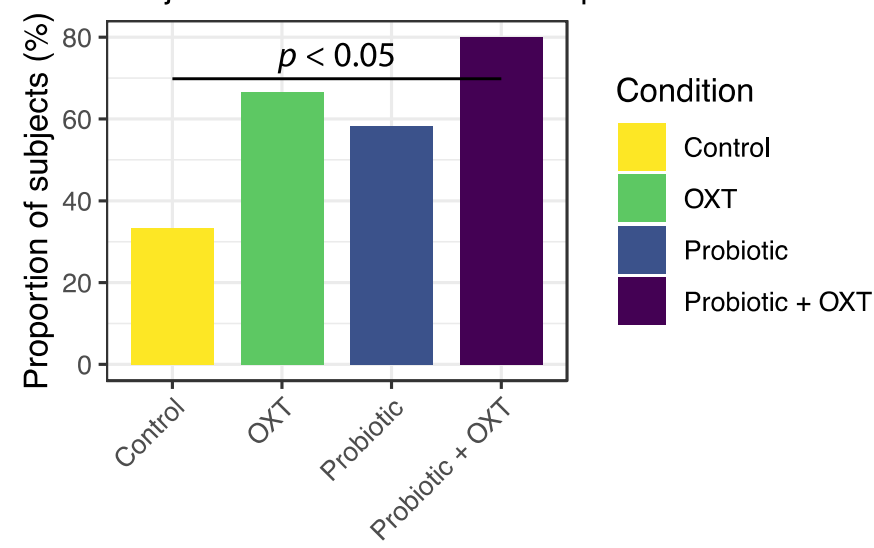

Figure 2. Proportion of subjects displaying improvement in CGI-I overtime among all subjects within a treatment condition. The z-test for equality of proportions is applied and the number of subjects displaying at least minimal improvement $(\mathrm{CGI}-\mathrm{I} \leq 3)$ in the control condition and the probiotic + OXT condition is significantly different $(p<0.05)$, while the changes of probiotic and OXT alone groups are non-significant, though a trend of improvement is seen in both intervention groups.

GIS showed no significant changes in the three treatment groups compared with the placebo group over the treatment course $(p>0.05)$.

\subsection{Gut Microbiome}

The gut microbiome was investigated by sequencing the fecal DNA. Although $\alpha$ - and $\beta$-diversity showed no significant changes in this study (Supp. Figure S2), we found a significant increase in microbiota hubs and numbers of connection edges uniquely at V3 as compared to the two previous visits V1 and V2 (Figure ??A), using a SparCC cutoff of 0.7. The lines or edges of the connections were significantly increased in both the OXT alone group ( $p<0.001)$ and the combination group $(p<0.005$, Figure ??B), however, the number of articulation points (those with halos around the node also called "hubs") were only significantly more in the combination group (Pearson's $\chi^{2}$-test with Yates continuity correction, $p<0.05$, Figure ??C).

When we investigated those key hub taxa with a hub score greater than 0.8 , interestingly, we found a distinct panel of hubs (marked as " + ") in the three treatment groups without overlaps. Christensenellaceae R7, Ruminococcaceae UCG-002, Lachnospiraceae UCG001, Blautia, and Barnesiella were only present in the combination therapy group; distinct hubs, Coprococcus-2, Rikenellaceae RC9, Bilophila, Catenibacterium, and Holdemanella, were only found in the OXT alone group; while Roseburia, Veillonella, and Streptococcus were only present in the probiotics group. None of the key hubs were only found in the placebo group (Supp. Table S1).

Functional gene predictive analysis indicated that several genes trended towards greater abundances in the combination group over the 28-week treatment period. Notably, genes encoding transporters, $\mathrm{ABC}$ transporters, transcription factors, sporulation, starch and sucrose metabolism, porphyrin and chlorophyll metabolism, signal transcription metabolism, arginine and proline metabolism, and thiamine metabolism were found to be more enriched in combination groups than the other groups, although the difference was not statistically significant ( $p>0.05$, Figure 4$)$.

We then performed the Spearman correlation analysis to assess the correlation between socio-behavioral parameters measured by the ABC and SRS and microbiota relative abundance at baseline and over the course of treatment. Interestingly, the taxa Eubacterium hallii group was found to be significantly associated with total scores $(R=-0.59$, false discovery rate-adjusted $P(F D R)=0.00767)$ and three subscales of the SRS before treatment (SRS communication: $\mathrm{R}=-0.55, \mathrm{FDR}=0.04282$ ); $\mathrm{SRS}$ mannerism: $\mathrm{R}=-0.6, \mathrm{FDR}=0.01753$; SRS motivation: $\mathrm{R}=-0.56, \mathrm{FDR}=0.0645$; Table 3 ); the strongest negative correlation 
was found between the Eubacterium hallii group and the SRS cognition score (Spearman's rho $=-0.97, p=0.0048$, FDR < 0.1). Furthermore, the absolute change (V3-V1) in Eubacterium hallii group abundance in the combination therapy group is positively correlated with the baseline SRS cognition score (Spearman's rho $=0.71, p=0.05$ ), meanwhile, the absolute change (V3-V1) in Rikenelaceae, Alistipes, Christensenellaceae R7, and Ruminococcaceae UCG-002 in the combination therapy group positively correlated with the ABC stereotypic behavior score at baseline (Table 4). Of note, Rikenelaceae and Alistipes were found to be significantly correlated with SRS motivation at baseline (Table 3), while Christensenellaceae R7 and Ruminococcaceae UCG-002 are two out of five important and unique hubs found only in the combination treatment group (Supp. Table S1). Additionally, Lachnospiraceae (uncultured) was found to be negatively correlated with the ABC inappropriate speech at baseline $(\mathrm{R}=-0.68, \mathrm{FDR}=0.04247)$.
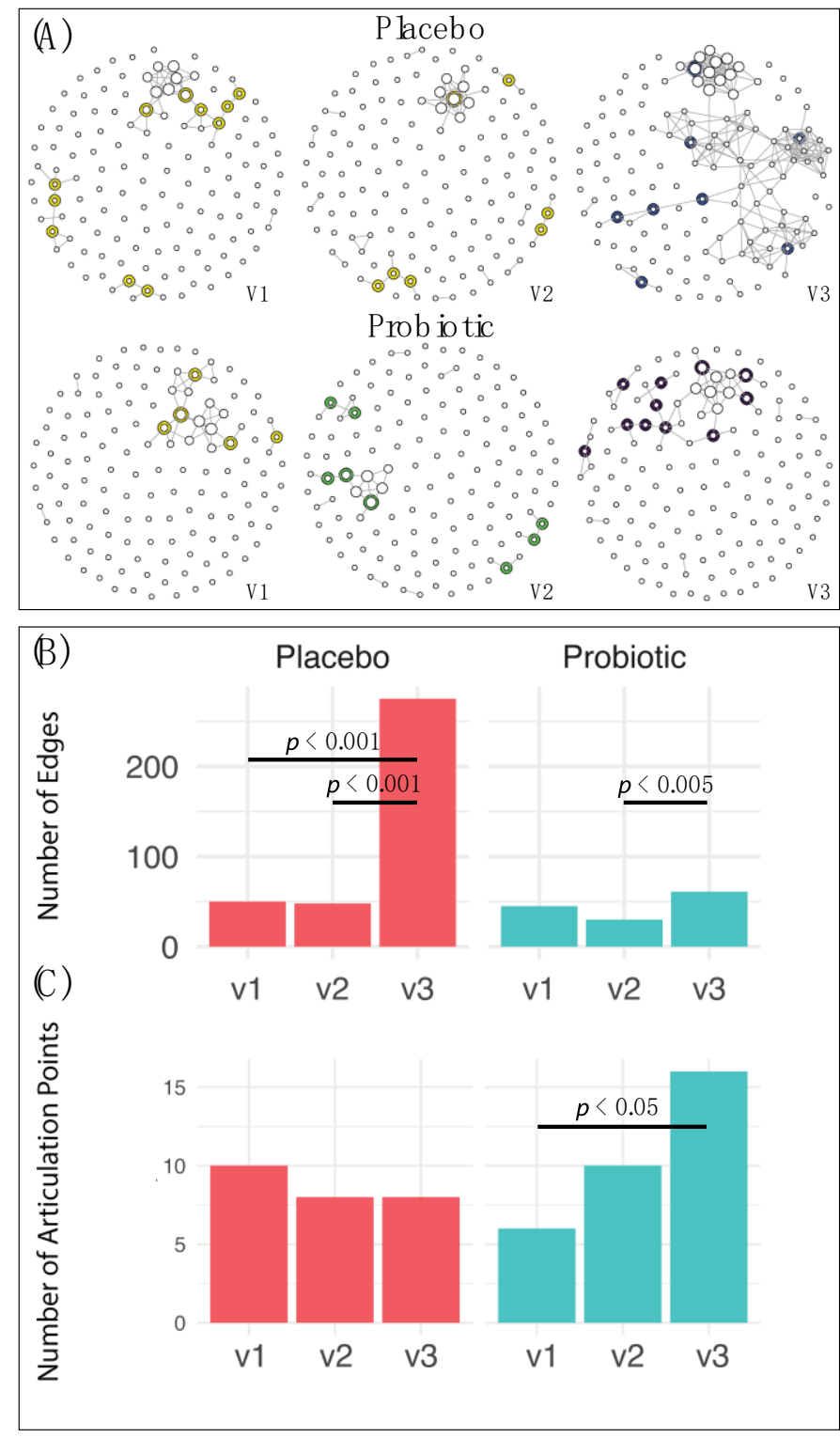

Figure 3. SparCC network associations between genus-level gut microbiota between subjects receiving placebo and those receiving the active probiotic overtime, using a SparCC cutoff of 0.7. Placebo group V1 is baseline, V2 is after placebo, V3 is after placebo added OXT; probiotics group V1 is baseline, V2 is after probiotics, V3 is after probiotics added OXT. (A) SparCC co-occurrence network. Articulation points are marked as halos around the node. Hub score is indicated by the size of the node. 
(B) The number of lines or edges is significantly enriched in both the OXT alone and combination groups at visit 3 (Pearson's $\chi^{2}$-test with Yates' continuity correction, $p<0.005$ ). (C) The number of articulation points is only significantly increased in the combination group at V3 compared to baseline number of articulation points (Pearson's $\chi^{2}$-test with Yates' continuity correction, $p<0.05$ ).

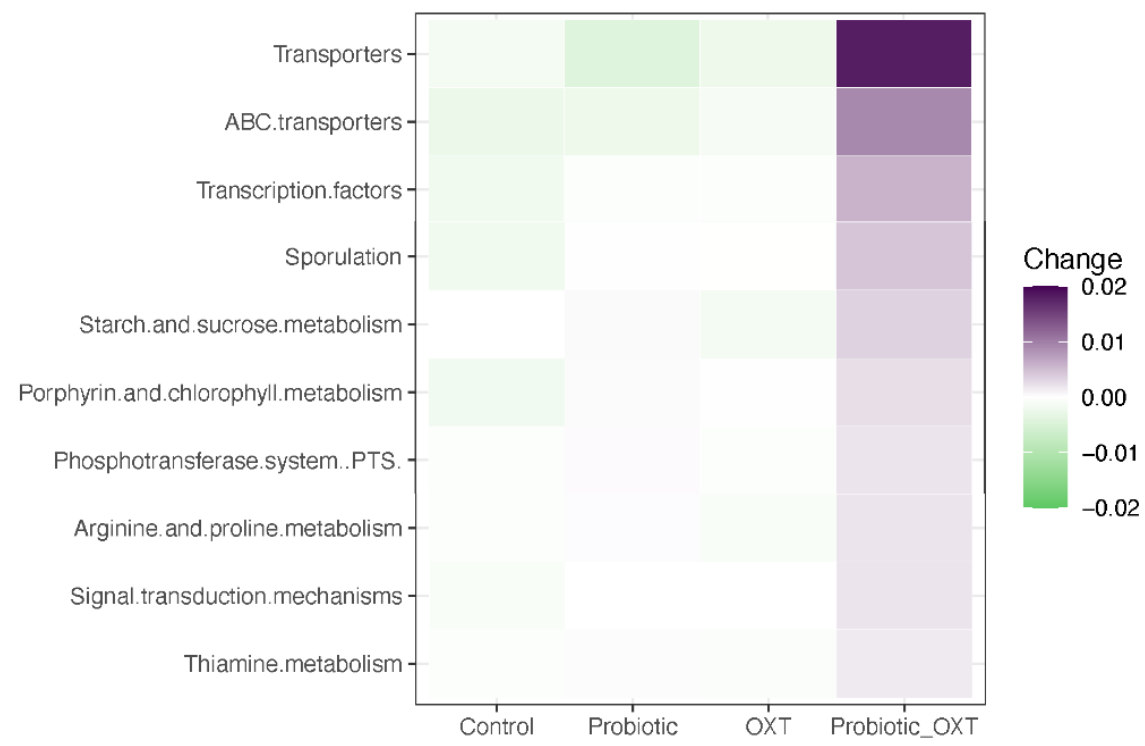

Figure 4. Heatmap of mean change in predicted functional profile based on gut microbiota abundance across four study groups. Shown changes in functional profiling indices demonstrated more changes in the combination group, although these changes are not significantly different when compared to the control group $(p>0.05)$.

Table 3. Spearman correlations between the microbiota relative abundance and socio-behavioral parameters before treatment for all subjects.

\begin{tabular}{llcc}
\hline \multicolumn{1}{c}{ Clinical Feature } & \multicolumn{1}{c}{ Microbiome Taxa } & \multicolumn{1}{c}{$\boldsymbol{R}$} & FDR $^{*}$ \\
\hline ABC Inappropriate Speech (T) & Lachnospiraceae (uncultured) & -0.68 & 0.04247 \\
SRS Communication (T) & Eubacterium hallii Group & -0.55 & 0.04282 \\
SRS Mannerisms (T) & Eubacterium hallii Group & -0.60 & 0.01753 \\
SRS Motivation (T) & Rikenellaceae & -0.58 & 0.0645 \\
& Alistipes & -0.58 & 0.0645 \\
& Eubacterium hallii Group & -0.56 & 0.0645 \\
SRS Total Score (T) & Eubacterium hallii Group & -0.59 & 0.00767 \\
\hline
\end{tabular}

* All presented correlations are significant at FDR $<0.1$.

Table 4. Significant correlations between primary outcomes and microbiota relative abundance in probiotic group and placebo group subjects based on Spearman's rank correlation.

\begin{tabular}{|c|c|c|c|c|c|}
\hline \multirow[t]{2}{*}{ Clinical Feature } & \multirow[t]{2}{*}{ Microbiome Taxa } & \multicolumn{2}{|c|}{$\begin{array}{c}\text { Probiotic Group } \\
(n=18)\end{array}$} & \multicolumn{2}{|c|}{$\begin{array}{l}\text { Placebo Group } \\
\quad(n=17)\end{array}$} \\
\hline & & $R$ & $p$-Value & $R$ & $p$-Value \\
\hline SRS Cognition T (V1) & Eubacterium hallii Group (V3-V1) & 0.71 & 0.050 & 0.01 & 0.790 \\
\hline SRS Cognition T (V3-V1) & Eubacterium hallii Group (V1) & -0.97 & 0.005 * & 0.17 & 0.750 \\
\hline \multirow[t]{4}{*}{ ABC Stereotypic Behavior T (V1) } & Rikenellaceae (V3-V1) & 0.94 & 0.017 & 0.20 & 0.590 \\
\hline & Alistipes (V3-V1) & 0.94 & 0.017 & -0.02 & 0.960 \\
\hline & Christensenellaceae R-7 Group (V3-V1) & 0.82 & 0.046 & -0.09 & 0.810 \\
\hline & Ruminococcaceae UCG-002 (V3-V1) & 0.83 & 0.058 & -0.37 & 0.290 \\
\hline
\end{tabular}

${ }^{*}$ FDR $<0.1$ based on screening of results. 


\subsection{Blood Serum Markers}

For the OXT level as measured, there were no significant changes of the four groups $(p>0.05)$ (Figure 5A). For the inflammatory markers tested in this study, a trend of greater decrease in S100 in the OXT alone group (Figure 5B) and IL-1 $\beta$ levels in the combination therapy group (Figure 5C) were observed; however, these differences were not statistically significant (Wilcoxon rank-sum test, $p>0.05$ ). By the Spearman correlation analysis, we also found that the S100 level positively correlated with ABC irritability (Figure 5D) and ABC hyperactivity/non-compliance scores (Figure 5D) at baseline.

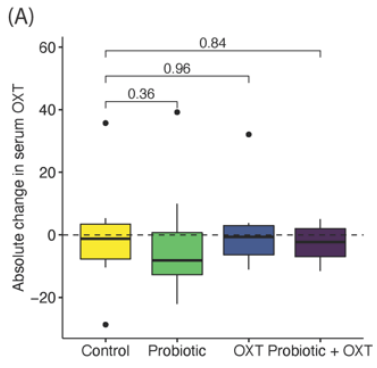

(D)
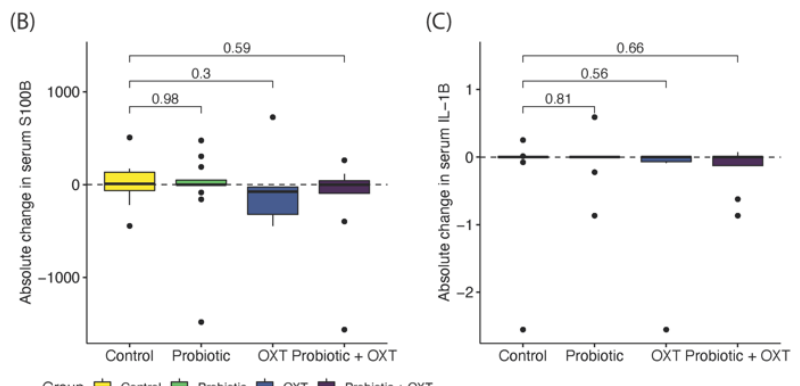

$\left(D^{\prime}\right)$

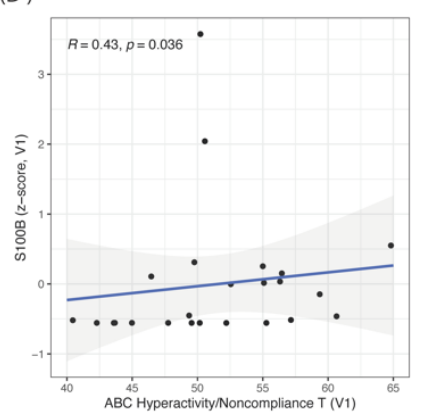

Figure 5. Summary of longitudinal serum marker changes and associated correlations. Absolute changes in serum OXT (A), S100B (B), and IL-1ß (C) levels, comparing each treatment group against controls. Baseline serum S100B is positively correlated with ABC irritability T (D) and ABC hyperactivity/noncompliance T-scores $\left(\mathbf{D}^{\prime}\right)$.

\section{Discussion}

In this pilot study, we explored and compared two promising interventions, probiotics and oxytocin, both alone and in combination, against placebo controls. All interventions were well tolerated, and no major adverse events were observed. Only in the combination treatment group, we observed a trend of improvement in social and behavioral measurements (ABC and SRS), particularly in the ABC total score $(p=0.077), \mathrm{ABC}$ stereotyped behavior sub-score $(p=0.069)$, and SRS cognition sub-score $(p=0.059)$. Meanwhile, a significant improvement of CGI was found only in the combination treatment group compared to the placebo, not in the probiotics or the OXT treatment alone groups. CGI provides a brief, stand-alone assessment of the clinician's view of the patient's global functioning prior to and after initiating a study medication. The CGI-I represents the change from the initiation of treatment on a seven-point scale [24]. In this study, the CGI-I was conducted by the clinician, who was totally blinded in treatment status and was also well acquainted with the subjects. Our finding that combination therapy elicited significant clinical improvement has not been reported previously. Previously, PS128 was found to increase dopamine and serotonin in different animal studies [12,13]; however, its relationship with oxytocin has not been tested. A potential mechanism of induction for increased serotonin secretion posits that bacterial tryptophan secretion catabolites may interact with intestinal enteroendocrine cells, thereby increasing intestinal motility and modulating the central nervous system (CNS) [28]. Furthermore, there is growing evidence for crucial interactions among the dopaminergic system, oxytocin/vasopressin, and serotoninergic systems in different areas 
of the brain that greatly influence human social behavior [29,30]. We believe that this finding not only opens a new avenue for ASD treatment but also furthers our knowledge about the gut-brain axis and ASD pathogenesis and warrants further studies.

Building on our finding of psychopathology improvement with combination therapy, we found some significant favorable changes in the gut microbiome over the intervention course. In particular, a significant increase in the SparCC co-occurrence network was found. The lines of the connections were significantly increased in both the OXT alone group $(p<0.001)$ and the combination group $(p<0.005)$, however, the number of articulation points (hubs) were only significantly more in the combination group $(p<0.05)$ not in the OXT alone group $(p>0.05)$, which suggests more critical and meaningful microbiome interactions are involved in the combination therapy. It is well known that an articulation point in a network is a node whose removal disconnects the network. This new finding favors the synergistic effects of the combination therapy. When examining the driving species of articulation points, we observed that the identified microbiota in the combination treatment group is unique not only from placebo group but also without overlaps with either the probiotics or the OXT alone group. Among those with a high hub score $(>0.8)$ in the combination therapy group, both Blautia and Barnesiella were previously reported to be reduced in the gut of ASD patients [31-33], and both can promote butyrate production, which benefits gut health [34]. Christensenellaceae R7, Ruminococcaceae UCG-002, and Lachnospiraceae UCG-001 have not yet been reported in ASD, but their health benefits related to weight, gut health, and diabetes have been reported. The enrichment of these hubs overall favors improving metabolism and inflammation [35-39]. The findings obtained from the OXT alone group with high score hubs of Bilophila, Coprococcus-2, Holdemanella, Rikenellaceae, and Catenibacterium also favor anti-inflammation and gut health in general [5,40-45]. Similarly, Roseburia, Veillonella, and Streptococcus, which were found to have high hub scores in the probiotics group, also promote anti-inflammation, gut health, and additionally, carbohydrate metabolism [5,40,46,47]. In the combination group, significantly increased numbers of the articulation points are likely contributing to their better treatment responses than each treatment alone; the distinct hub panel from the single therapy groups also supports the synergistic effects as observed in the combination therapy group. The advance of network theory helps to disentangle the higher order interactions that occur within microbiomes [48], which could be more important than the microbiome diversity representation.

Additionally, predicted functional gene analysis suggested that several important pathways are more activated in the combination group than the placebo and other treatment groups. The pathways with the highest discriminative power in the combination group were "Transporters" followed by "ABC transporters", transcription factors, starch and sucrose metabolism, arginine and proline metabolism, and thiamine metabolism. ABC transporters couple energy metabolism and mediate the uptake of nutrients and physiological functions, which were found to be repressed in an ASD model with impairment of the neuronal network $[49,50]$. The starch and sucrose metabolism pathways have been found to be down-regulated in ASD [51]. The human gut microbiome is a critical component of digestion, as it facilitates the breakdown of complex carbohydrates and proteins [52]. Arginine has been shown to be substantially reduced in cases of gut inflammation and infection [53]. As a metabolic precursor for nitric oxide (NO), it regulates neuron survival, differentiation, synaptic activity, and plasticity [54]. Thiamine (vitamin $B_{1}$ ) is an essential cofactor that when deficient, contributes to symptoms such as confusion, reduced memory, and sleep disturbances [55], and when adequately concentrated, promotes homeostasis of a healthy gut ecosystem. These favorable findings further supported the use of combination therapy as a promising treatment approach than using them alone, as a synergistic effect was involved to facilitate energy metabolism and normal physiological functions.

Importantly, gut microbiome was found to be highly correlated with social behavioral parameters. Eubacterium hallii was found to be significantly negatively correlated with the SRS total score and sub-scores, particularly the SRS cognition sub-score. More enriched 
gut Eubacterium hallii abundances correlate with lower SRS scores, thus representing a better social function level. This strong correlation is not only observed at baseline but also with the absolute increase in the combination group at visit three. As mentioned earlier, the improvement of the SRS cognition subscale in the combination group, as shown in Table $2(p=0.059)$, is one of the most prominent trends of improvement observed. The higher the Eubacterium hallii at baseline, the more favorable the improvement of social cognition over the course of the combination treatment. The lower the level of social cognition (with a higher score) at baseline, the more an increase in Eubacterium hallii in the combination group was observed. Eubacterium hallii can utilize glucose, and the fermentation intermediates acetate and lactate to form butyrate, which benefits gut health [56,57]; however, this promising taxon has not yet been reported in ASD patients. Additionally, Christensenellaceae R7 and Ruminococcaceae UCG-002, two of the five unique hubs that were only observed in the combination treatment group (V3-V1), were found to be positively correlated with the $\mathrm{ABC}$ stereotypic behavior sub-score, which describes one of the ASD core symptoms. This correlation analysis further demonstrates the strong association of gut microbiome with ASD core symptoms at baseline and after a favorable treatment response in the combination group.

To further our knowledge of these treatment responses, we also measured serum oxytocin and inflammatory markers over the course of the treatment. In this study, we did not find significant changes of oxytocin level in the three treatment groups when compared with the placebo group.

The aberrant OXT serum levels have been reported in ASD individuals to varying degrees, sometimes decreased [58,59], sometimes no difference [60,61], and sometimes enriched compared to non-ASD controls [62]. These differences could be related to subsets of the ASD population with reduced biosynthesis or release of OXT [63,64], dysfunctional OXT processing dysfunction, or oxytocin receptor abnormalities [65]. Further studies are warranted to investigate these potential ASD subtypes and to resolve these variable results and treatment responses in different subsets.

Inflammatory mechanisms linked with ASD have been widely reported. Inflammatory cytokines were found to be significantly elevated in ASD individuals compared with healthy controls [66,67]. Similarly, brain injury and inflammatory markers, GFAP, MBP, and S100B, have been found to be significantly enriched in ASD children than controls [67-71]; these brain injury markers and cytokine release subsequently trigger glial cell activation and the inflammatory process in the brain [72]. In this study, we tested these four serum inflammatory markers and found that a trend of decrease in S100 in the OXT group, and the decrease in IL-1 $\beta$ to be more pronounced in combination treatment. S100B was shown to have a significant positive correlation with the severity of problem behaviors ( $A B C$ irritability and hyperactivity scores at baseline; $p<0.05$ ).

There are several limitations of the study that deserve consideration. (1) Despite our adoption of proper recruitment and retention strategies, the participant enrollment and retention for this trial were challenging. A relatively small sample size in this pilot trial limited the statistical power and further subgroup analysis. (2) Although there was no statistical difference in clinical indices between the probiotics and placebo groups at baseline, the wide age range used in this study resulted in high subject population heterogeneity and potentially variable treatment efficacy. Future studies with a larger sample size and subgroup stratification are warranted. (3) Due to considerable Asian and other minority patients with some cultural and language barriers, in addition to multiple influencing factors on behavioral variabilities, the parent rating of social behavioral scales may be somewhat biased. (4) Sequential comparisons were not made at the same time point for the four intervention groups. The two-stage design seems inferior to simply having four groups with a $2 \times 2$ factorial design; in this design, the prolonged treatment course might be influenced by other randomly occurring factors. 


\section{Conclusions}

In the present pilot trial, we demonstrate that the concurrent supplementation of oral probiotic Lactobacillus plantarum PS128 and intranasal OXT in participants with ASD may reduce ASD core socio-behavioral symptoms and clinical global functioning. Statistically significant improvements in ASD-related outcomes over the treatment course via combined therapy are attributed to the proposed synergistic interactions between the two treatments, which are mediated via the gut-brain axis. Furthermore, participants receiving combined therapy showed significant improvements in gut microbiome dysbiosis characterized by several distinct hub networks. Despite such promising preliminary findings, the underlying mechanisms and causal relationships of such synergistic effects remain elusive and deserve further investigation in large-scale and well-designed trials.

Supplementary Materials: The following are available online at https://www.mdpi.com/article/ 10.3390/nu13051552/s1, Figure S1: Waterfall plot of reduction in CGI-S score for each subject in different intervention groups, Figure S2: Overview of gut microbiome species diversity, Table S1: Summary of identified key hub taxa based on SparCC network analysis.

Author Contributions: Conceptualization, X.-J.K.; methodology, X.-J.K.; software, K.L.; validation, H.L. and Y.S.; formal analysis, K.L.; investigation, X.-J.K., J.L., M.K., H.S., P.S., S.L., K.L., J.W., M.F., M.L., V.H., T.D., R.T., S.-T.C., L.X., C.C., K.K., and A.K.; resources, X.-J.K.; data curation, R.T., S.L., and K.L.; writing—original draft preparation, X.-J.K.; writing—review and editing, X.-J.K., K.L., C.C., A.L., M.-S.J., H.S., and J.L.; visualization, K.L. and M.F.; supervision, X.-J.K.; project administration, X.-J.K. and S.L.; funding acquisition, X.-J.K. All authors have read and agreed to the published version of the manuscript.

Funding: This research was funded by Massachusetts General Hospital, grant numbers \#230361 and \#233263.

Institutional Review Board Statement: The study was conducted according to the guidelines of the Declaration of Helsinki and approved by the Institutional Review Board of Massachusetts General Hospital (2017P001667, 13 July 2018).

Informed Consent Statement: Informed consent was obtained from all subjects involved in the study.

Data Availability Statement: The data presented in this study are available on request from the corresponding author.

Acknowledgments: We sincerely thank Bruce Rosen for his valuable advice and great support throughout the course of the study. We thank Yen-Wenn Liu for her generous help in the blinded microbiome analysis. We thank Steven Stufflebeam for his generous clinical service and assistance during the project. We thank Pamela Richtmyer for her kind help on IND and regulatory guidance. We thank the GenoInfo Core Facility (C1) funded by National Core Facility for Biopharmaceuticals of MOST Taiwan (MOST108-2319-B-010-001) for assisting the blinded microbiome data analysis. We would also like to thank Bened Biomedical Co., Ltd for providing the PS128 and placebo products as a free gift.

Conflicts of Interest: The authors declare no conflict of interest. Both the funding sources and the probiotic and placebo product provider had no role in the design of the study; in the collection, analyses, or interpretation of data; in the writing of the manuscript, or in the decision to publish the esults.

\section{References}

1. Maenner, M.J.; Shaw, K.A.; Baio, J.; Washington, A.; Patrick, M.; DiRienzo, M.; Christensen, D.L.; Wiggins, L.D.; Pettygrove, S.; Andrews, J.G.; et al. Prevalence of Autism Spectrum Disorder Among Children Aged 8 Years-Autism and Developmental Disabilities Monitoring Network, 11 Sites, United States, 2016. MMWR Surveill. Summ. 2020, 69, 1-12. [CrossRef]

2. Alam, R.; Abdolmaleky, H.M.; Zhou, J.-R. Microbiome, inflammation, epigenetic alterations, and mental diseases. Am. J. Med Genet. Part B: Neuropsychiatr. Genet. 2017, 174, 651-660. [CrossRef]

3. Fung, T.C.; A Olson, C.; Hsiao, T.C.F.C.A.O.E.Y. Interactions between the microbiota, immune and nervous systems in health and disease. Nat. Neurosci. 2017, 20, 145-155. [CrossRef] [PubMed] 
4. Kong, X.; Liu, J.; Cetinbas, M.; Sadreyev, R.; Koh, M.; Huang, H.; Adeseye, A.; He, P.; Zhu, J.; Russell, H.; et al. New and Preliminary Evidence on Altered Oral and Gut Microbiota in Individuals with Autism Spectrum Disorder (ASD): Implications for ASD Diagnosis and Subtyping Based on Microbial Biomarkers. Nutr. 2019, 11, 2128. [CrossRef] [PubMed]

5. Liu, Y.-W.; Liong, M.T.; Chung, Y.-C.E.; Huang, H.-Y.; Peng, W.-S.; Cheng, Y.-F.; Lin, Y.-S.; Wu, Y.-Y.; Tsai, Y.-C. Effects of Lactobacillus plantarum PS128 on Children with Autism Spectrum Disorder in Taiwan: A Randomized, Double-Blind, PlaceboControlled Trial. Nutr. 2019, 11, 820. [CrossRef]

6. Kong, X.-J.; Liu, J.; Li, J.; Kwong, K.; Koh, M.; Sukijthamapan, P.; Guo, J.J.; Sun, Z.J.; Song, Y. Probiotics and oxytocin nasal spray as neuro-social-behavioral interventions for patients with autism spectrum disorders: A pilot randomized controlled trial protocol. Pilot Feasibility Stud. 2020, 6, 1-9. [CrossRef] [PubMed]

7. Dinan, T.G.; Stanton, C.; Cryan, J.F. Psychobiotics: A Novel Class of Psychotropic. Biol. Psychiatry 2013, 74, 720-726. [CrossRef]

8. Desbonnet, L.; Garrett, L.; Clarke, G.; Bienenstock, J.; Dinan, T.G. The probiotic Bifidobacteria infantis: An assessment of potential antidepressant properties in the rat. J. Psychiatr. Res. 2008, 43, 164-174. [CrossRef]

9. Savignac, H.M.; Kiely, B.; Dinan, T.G.; Cryan, J.F. Bifidobacteriaexert strain-specific effects on stress-related behavior and physiology in BALB/c mice. Neurogastroenterol. Motil. 2014, 26, 1615-1627. [CrossRef]

10. Davari, S.; Talaei, S.A.; Alaei, H.; Salami, M. Probiotics treatment improves diabetes-induced impairment of synaptic activity and cognitive function: Behavioral and electrophysiological proofs for microbiome-gut-brain axis. Neuroscience 2013, 240, 287-296. [CrossRef]

11. Hsiao, E.Y.; McBride, S.W.; Hsien, S.; Sharon, G.; Hyde, E.R.; McCue, T.; Codelli, J.A.; Chow, J.; Reisman, S.E.; Petrosino, J.F.; et al. Microbiota Modulate Behavioral and Physiological Abnormalities Associated with Neurodevelopmental Disorders. Cell 2013, 155, 1451-1463. [CrossRef]

12. Liu, Y.-W.; Liu, W.-H.; Wu, C.-C.; Juan, Y.-C.; Wu, Y.-C.; Tsai, H.-P.; Wang, S.; Tsai, Y.-C. Psychotropic effects of Lactobacillus plantarum PS128 in early life-stressed and naïve adult mice. Brain Res. 2016, 1631, 1-12. [CrossRef]

13. Liu, W.-H.; Chuang, H.-L.; Huang, Y.-T.; Wu, C.-C.; Chou, G.-T.; Wang, S.; Tsai, Y.-C. Alteration of behavior and monoamine levels attributable to Lactobacillus plantarum PS128 in germ-free mice. Behav. Brain Res. 2016, 298, 202-209. [CrossRef] [PubMed]

14. Erdman, S.; Poutahidis, T. Microbes and Oxytocin. International Review of Neurobiology 2016, 131, 91-126. [CrossRef] [PubMed]

15. Pobbe, R.L.; Pearson, B.L.; Defensor, E.B.; Bolivar, V.J.; Young, W.S.; Lee, H.-J.; Blanchard, D.C.; Blanchard, R.J. Oxytocin receptor knockout mice display deficits in the expression of autism-related behaviors. Horm. Behav. 2012, 61, 436-444. [CrossRef]

16. Teng, B.L.; Nikolova, V.D.; Riddick, N.V.; Agster, K.L.; Crowley, J.J.; Baker, L.K.; Koller, B.H.; Pedersen, C.A.; Jarstfer, M.B.; Moy, S.S. Reversal of social deficits by subchronic oxytocin in two autism mouse models. Neuropharmacol. 2016, 105, 61-71. [CrossRef] [PubMed]

17. Chao, S.-H.; Wu, R.-J.; Watanabe, K.; Tsai, Y.-C. Diversity of lactic acid bacteria in suan-tsai and fu-tsai, traditional fermented mustard products of Taiwan. Int. J. Food Microbiol. 2009, 135, 203-210. [CrossRef]

18. Liu, W.-H.; Yang, C.-H.; Lin, C.-T.; Li, S.-W.; Cheng, W.-S.; Jiang, Y.-P.; Wu, C.-C.; Chang, C.-H.; Tsai, Y.-C. Genome architecture of Lactobacillus plantarum PS128, a probiotic strain with potential immunomodulatory activity. Gut Pathog. 2015, 7, 22. [CrossRef]

19. Liao, P.-L.; Wu, C.-C.; Chen, T.-Y.; Tsai, Y.-C.; Peng, W.-S.; Yang, D.-J.; Kang, J.-J. Toxicity Studies of Lactobacillus plantarum PS128TM Isolated from Spontaneously Fermented Mustard Greens. Foods 2019, 8, 668. [CrossRef] [PubMed]

20. Yatawara, C.J.; Einfeld, S.L.; Hickie, I.B.; A Davenport, T.; Guastella, A.J. The effect of oxytocin nasal spray on social interaction deficits observed in young children with autism: A randomized clinical crossover trial. Mol. Psychiatry 2016, 21, 1225-1231. [CrossRef]

21. Parker, K.J.; Oztan, O.; Libove, R.A.; Sumiyoshi, R.D.; Jackson, L.P.; Karhson, D.S.; Summers, J.E.; Hinman, K.E.; Motonaga, K.S.; Phillips, J.M.; et al. Intranasal oxytocin treatment for social deficits and biomarkers of response in children with autism. Proc. Natl. Acad. Sci. USA 2017, 114, 8119-8124. [CrossRef] [PubMed]

22. Constantino, J.; Gruber, C. Social Responsive Scale (SRS) Manual; Western Psychological Services: Los Angeles, CA, USA, 2005.

23. Aman, M.G.; Singh, N.N.; Stewart, A.W.; Field, C.J. The Aberrant Behavior Checklist: A Behavior Rating Scale for the As-sessment of Treatment Effects. Am. J. Ment. Defic. 1985, 89, 485-491. [PubMed]

24. Busner, J.; Targum, S.D. The Clinical Global Impressions Scale: Applying a Research Tool in Clinical Practice. Psychiatry (Edgmont) 2007, 4, 28-37.

25. Bolyen, E.; Rideout, J.R.; Dillon, M.R.; Bokulich, N.A.; Abnet, C.C.; Al-Ghalith, G.A.; Alexander, H.; Alm, E.J.; Arumugam, M.; Asnicar, F.; et al. Reproducible, interactive, scalable and extensible microbiome data science using QIIME 2. Nat. Biotechnol. 2019, 37, 852-857. [CrossRef] [PubMed]

26. Friedman, J.; Alm, E.J. Inferring Correlation Networks from Genomic Survey Data. PLoS Comput. Biol. 2012, 8, e1002687. [CrossRef] [PubMed]

27. Mallick, H.; Rahnavard, A.; McIver, L.J.; Ma, S.; Zhang, Y.; Nguyen, L.H.; Tickle, T.L.; Weingart, G.; Ren, B.; Schwager, E.H.; et al. Multivariable Association Discovery in Population-Scale Meta-Omics Studies. bioRxiv 2021. [CrossRef]

28. Ye, L.; Bae, M.; Cassilly, C.D.; Jabba, S.V.; Thorpe, D.W.; Martin, A.M.; Lu, H.-Y.; Wang, J.; Thompson, J.D.; Lickwar, C.R.; et al. Enteroendocrine cells sense bacterial tryptophan catabolites to activate enteric and vagal neuronal pathways. Cell Host Microbe 2021, 29, 179-196.e9. [CrossRef] [PubMed]

29. Skuse, D.H.; Gallagher, L. Dopaminergic-neuropeptide interactions in the social brain. Trends Cogn. Sci. 2009, 13, 27-35. [CrossRef] 
30. Marotta, R.; Risoleo, M.C.; Messina, G.; Parisi, L.; Carotenuto, M.; Vetri, L.; Roccella, M. The Neurochemistry of Autism. Brain Sci. 2020, 10, 163. [CrossRef]

31. Inoue, R.; Sakaue, Y.; Sawai, C.; Sawai, T.; Ozeki, M.; Romero-Pérez, G.A.; Tsukahara, T. A preliminary investigation on the relationship between gut microbiota and gene expressions in peripheral mononuclear cells of infants with autism spectrum disorders. Biosci. Biotechnol. Biochem. 2016, 80, 2450-2458. [CrossRef] [PubMed]

32. Luna, R.A.; Oezguen, N.; Balderas, M.; Venkatachalam, A.; Runge, J.K.; Versalovic, J.; Veenstra-VanderWeele, J.; Anderson, G.M.; Savidge, T.; Williams, K.C. Distinct Microbiome-Neuroimmune Signatures Correlate With Functional Abdominal Pain in Children With Autism Spectrum Disorder. Cell. Mol. Gastroenterol. Hepatol. 2017, 3, 218-230. [CrossRef] [PubMed]

33. Averina, O.V.; Kovtun, A.S.; Polyakova, S.I.; Savilova, A.M.; Rebrikov, D.V.; Danilenko, V.N. The bacterial neurometabolic signature of the gut microbiota of young children with autism spectrum disorders. J. Med Microbiol. 2020, 69, 558-571. [CrossRef]

34. Lustgarten, M.S. The Role of the Gut Microbiome on Skeletal Muscle Mass and Physical Function: 2019 Update. Front. Physiol. 2019, 10, 1435. [CrossRef] [PubMed]

35. Garcia-Mantrana, I.; Selma-Royo, M.; Alcantara, C.; Collado, M.C. Shifts on Gut Microbiota Associated to Mediterranean Diet Adherence and Specific Dietary Intakes on General Adult Population. Front. Microbiol. 2018, 9, 890. [CrossRef] [PubMed]

36. Wang, Y.; Zhang, H.; Zhu, L.; Xu, Y.; Liu, N.; Sun, X.; Hu, L.; Huang, H.; Wei, K.; Zhu, R. Dynamic Distribution of Gut Microbiota in Goats at Different Ages and Health States. Front. Microbiol. 2018, 9, 2509. [CrossRef]

37. Guo, M.; Li, Z. Polysaccharides isolated fromNostoc communeVaucher inhibit colitis-associated colon tumorigenesis in mice and modulate gut microbiota. Food Funct. 2019, 10, 6873-6881. [CrossRef]

38. Gao, B.; Zhong, M.; Shen, Q.; Wu, Y.; Cao, M.; Ju, S.; Chen, L. Gut microbiota in early pregnancy among women with Hyperglycaemia vs. Normal blood glucose. BMC Pregnancy Childbirth 2020, 20, 1-11. [CrossRef]

39. Ma, S.; You, Y.; Huang, L.; Long, S.; Zhang, J.; Guo, C.; Zhang, N.; Wu, X.; Xiao, Y.; Tan, H. Alterations in Gut Microbiota of Gestational Diabetes Patients During the First Trimester of Pregnancy. Front. Cell. Infect. Microbiol. 2020, 10, 58. [CrossRef] [PubMed]

40. Strati, F.; Cavalieri, D.; Albanese, D.; De Felice, C.; Donati, C.; Hayek, J.; Jousson, O.; Leoncini, S.; Renzi, D.; Calabrò, A.; et al. New evidences on the altered gut microbiota in autism spectrum disorders. Microbiome 2017, 5, 1-11. [CrossRef]

41. Tomova, A.; Soltys, K.; Kemenyova, P.; Karhanek, M.; Babinska, K. The Influence of Food Intake Specificity in Children with Autism on Gut Microbiota. Int. J. Mol. Sci. 2020, 21, 2797. [CrossRef]

42. Berding, K.; Donovan, S.M. Diet Can Impact Microbiota Composition in Children with Autism Spectrum Disorder. Front. Neurosci. 2018, 12, 515. [CrossRef]

43. El Hage, R.; Hernandez-Sanabria, E.; Arroyo, M.C.; Props, R.; Van De Wiele, T. Propionate-Producing Consortium Restores Antibiotic-Induced Dysbiosis in a Dynamic in vitro Model of the Human Intestinal Microbial Ecosystem. Front. Microbiol. 2019, 10, 1206. [CrossRef] [PubMed]

44. Kang, D.-W.; Park, J.G.; Ilhan, Z.E.; Wallstrom, G.; LaBaer, J.; Adams, J.B.; Krajmalnik-Brown, R. Reduced Incidence of Prevotella and Other Fermenters in Intestinal Microflora of Autistic Children. PLOS ONE 2013, 8, e68322. [CrossRef] [PubMed]

45. Jarett, J.K.; Carlson, A.; Serao, M.R.; Strickland, J.; Serfilippi, L.; Ganz, H.H. Diets with and without edible cricket support a similar level of diversity in the gut microbiome of dogs. PeerJ 2019, 7, e7661. [CrossRef] [PubMed]

46. Zhang, M.; Ma, W.; Zhang, J.; He, Y.; Wang, J. Analysis of gut microbiota profiles and microbe-disease associations in children with autism spectrum disorders in China. Sci. Rep. 2018, 8, 1-9. [CrossRef]

47. De Angelis, M.; Francavilla, R.; Piccolo, M.; De Giacomo, A.; Gobbetti, M. Autism spectrum disorders and intestinal microbiota. Gut Microbes 2015, 6, 207-213. [CrossRef]

48. Layeghifard, M.; Hwang, D.M.; Guttman, D.S. Disentangling Interactions in the Microbiome: A Network Perspective. Trends Microbiol. 2017, 25, 217-228. [CrossRef]

49. Liu, F.; Horton-Sparks, K.; Hull, V.; Li, R.W.; Martínez-Cerdeño, V. The valproic acid rat model of autism presents with gut bacterial dysbiosis similar to that in human autism. Mol. Autism 2018, 9, 1-13. [CrossRef]

50. Iritani, S.; Torii, Y.; Habuchi, C.; Sekiguchi, H.; Fujishiro, H.; Yoshida, M.; Go, Y.; Iriki, A.; Isoda, M.; Ozaki, N. The neuropathological investigation of the brain in a monkey model of autism spectrum disorder with ABCA13 deletion. Int. J. Dev. Neurosci. 2018, 71, 130-139. [CrossRef]

51. Rose, D.R.; Yang, H.; Serena, G.; Sturgeon, C.; Ma, B.; Careaga, M.; Hughes, H.K.; Angkustsiri, K.; Rose, M.; Hertz-Picciotto, I. Differential Immune Responses and Microbiota Profiles in Children with Autism Spectrum Disorders and Co-Morbid Gastrointestinal Symptoms. Brain Behav. Immun. 2018, 70, 354-368. [CrossRef]

52. Oliphant, K.; Allen-Vercoe, E. Macronutrient metabolism by the human gut microbiome: Major fermentation by-products and their impact on host health. Microbiome 2019, 7, 1-15. [CrossRef]

53. Fritz, J.H. Arginine Cools the Inflamed Gut. Infect. Immun. 2013, 81, 3500-3502. [CrossRef] [PubMed]

54. Tripathi, M.K.; Kartawy, M.; Amal, H. The role of nitric oxide in brain disorders: Autism spectrum disorder and other psychiatric, neurological, and neurodegenerative disorders. Redox Biol. 2020, 34, 101567. [CrossRef] [PubMed]

55. Dhir, S.; Tarasenko, M.; Napoli, E.; Giulivi, C. Neurological, Psychiatric, and Biochemical Aspects of Thiamine Deficiency in Children and Adults. Front. Psychiatry 2019, 10, 207. [CrossRef] [PubMed] 
56. Cao, X.; Liu, K.; Liu, J.; Liu, Y.-W.; Xu, L.; Wang, H.; Zhu, Y.; Wang, P.; Li, Z.; Wen, J.; et al. Dysbiotic Gut Microbiota and Dysregulation of Cytokine Profile in Children and Teens With Autism Spectrum Disorder. Front. Neurosci. $2021,15,635925$. [CrossRef]

57. Udayappan, S.; Manneras-Holm, L.; Chaplin-Scott, A.; Belzer, C.; Herrema, H.; Dallinga-Thie, G.M.; Duncan, S.H.; Stroes, E.S.G.; Groen, A.K.; Flint, H.J.; et al. Oral treatment with Eubacterium hallii improves insulin sensitivity in db/db mice. npj Biofilms Microbiomes 2016, 2, 16009. [CrossRef]

58. Husarova, V.M.; Lakatosova, S.; Pivovarciova, A.; Babinska, K.; Bakos, J.; Durdiakova, J.; Kubranska, A.; Ondrejka, I.; Ostatnikova, D. Plasma Oxytocin in Children with Autism and Its Correlations with Behavioral Parameters in Children and Parents. Psychiatry Investig. 2016, 13, 174-183. [CrossRef] [PubMed]

59. Zhang, H.-F.; Dai, Y.-C.; Wu, J.; Jia, M.-X.; Zhang, J.-S.; Shou, X.-J.; Han, S.-P.; Zhang, R.; Han, J.-S. Plasma Oxytocin and Arginine-Vasopressin Levels in Children with Autism Spectrum Disorder in China: Associations with Symptoms. Neurosci. Bull. 2016, 32, 423-432. [CrossRef]

60. Miller, M.; Bales, K.L.; Taylor, S.L.; Yoon, J.; Hostetler, C.M.; Carter, C.S.; Solomon, M. Oxytocin and Vasopressin in Children and Adolescents With Autism Spectrum Disorders: Sex Differences and Associations With Symptoms. Autism Res. 2013, 6, 91-102. [CrossRef] [PubMed]

61. Parker, K.J.; Garner, J.P.; Libove, R.A.; Hyde, S.A.; Hornbeak, K.B.; Carson, D.S.; Liao, C.-P.; Phillips, J.M.; Hallmayer, J.F.; Hardan, A.Y. Plasma oxytocin concentrations and OXTR polymorphisms predict social impairments in children with and without autism spectrum disorder. Proc. Natl. Acad. Sci. USA 2014, 111, 12258-12263. [CrossRef]

62. Jansen, L.M.C.; Wied, C.C.G.-D.; Wiegant, V.M.; Westenberg, H.G.M.; Lahuis, B.E.; Van Engeland, H. Autonomic and Neuroendocrine Responses to a Psychosocial Stressor in Adults with Autistic Spectrum Disorder. J. Autism Dev. Disord. 2006, 36, 891-899. [CrossRef]

63. Dadds, M.R.; Moul, C.; Cauchi, A.; Dobson-Stone, C.; Hawes, D.J.; Brennan, J.; Ebstein, R.E. Methylation of the oxytocin receptor gene and oxytocin blood levels in the development of psychopathy. Dev. Psychopathol. 2013, 26, 33-40. [CrossRef] [PubMed]

64. Jin, D.; Liu, H.-X.; Hirai, H.; Torashima, T.; Nagai, T.; Lopatina, O.; Shnayder, N.A.; Yamada, K.; Noda, M.; Seike, T.; et al. CD38 is critical for social behaviour by regulating oxytocin secretion. Nat. Cell Biol. 2007, 446, 41-45. [CrossRef] [PubMed]

65. Watanabe, T.; Otowa, T.; Abe, O.; Kuwabara, H.; Aoki, Y.; Natsubori, T.; Takao, H.; Kakiuchi, C.; Kondo, K.; Ikeda, M.; et al. Oxytocin receptor gene variations predict neural and behavioral response to oxytocin in autism. Soc. Cogn. Affect. Neurosci. 2016, 12, 496-506. [CrossRef]

66. Masi, A.; Quintana, D.S.; Glozier, N.; Lloyd, A.R.; Hickie, I.B.; Guastella, A.J. Cytokine aberrations in autism spectrum disorder: A systematic review and meta-analysis. Mol. Psychiatry 2014, 20, 440-446. [CrossRef] [PubMed]

67. Guloksuz, S.A.; Abali, O.; Cetin, E.A.; Gazioglu, S.B.; Deniz, G.; Yildirim, A.; Kawikova, I.; Guloksuz, S.; Leckman, J.F. Elevated plasma concentrations of S100 calcium-binding protein B and tumor necrosis factor alpha in children with autism spectrum disorders. Rev. Bras. Psiquiatr. 2017, 39, 195-200. [CrossRef]

68. Abou-Donia, M.B.; Suliman, H.B.; Siniscalco, D.; Antonucci, N.; Elkafrawy, P. De novo Blood Biomarkers in Autism: Autoantibodies against Neuronal and Glial Proteins. Behav. Sci. 2019, 9, 47. [CrossRef] [PubMed]

69. Esnafoglu, E.; Ayyıldız, S.N.; Cırrık, S.; Erturk, E.Y.; Erdil, A.; Daglı, A.; Noyan, T. Evaluation of serum Neuron-specific enolase, $\mathrm{S} 100 \mathrm{~B}$, myelin basic protein and glial fibrilliary acidic protein as brain specific proteins in children with autism spectrum disorder. Int. J. Dev. Neurosci. 2017, 61, 86-91. [CrossRef] [PubMed]

70. Mostafa, G.A.; Al-Ayadhi, L.Y. A lack of association between hyperserotonemia and the increased frequency of serum anti-myelin basic protein auto-antibodies in autistic children. J. Neuroinflamm. 2011, 8, 71. [CrossRef]

71. Gonzalez-Gronow, M.; Cuchacovich, M.; Francos, R.; Cuchacovich, S.; Blanco, A.; Sandoval, R.; Gomez, C.F.; Valenzuela, J.A.; Ray, R.; Pizzo, S.V. Catalytic autoantibodies against myelin basic protein (MBP) isolated from serum of autistic children impair in vitro models of synaptic plasticity in rat hippocampus. J. Neuroimmunol. 2015, 287, 1-8. [CrossRef]

72. Kern, J.K.; Geier, D.A.; Sykes, L.K.; Geier, M.R. Relevance of Neuroinflammation and Encephalitis in Autism. Front. Cell. Neurosci. 2016, 9, 519. [CrossRef] [PubMed] 